\title{
Formes du savoir, sens du savoir
}

L'exemple du Loup et l'Agneau de Jean de La Fontaine en classe de CE1 de l'école primaire française

Shapes of Knowledge, Sense of Knowledge. The Wolf and the Lamb by Jean de la Fontaine in CE1 Class Session (French Primary School)

\section{Luc Maisonneuve}

\section{(2) OpenEdition}

\section{Journals}

Édition électronique

URL : http://journals.openedition.org/pratiques/2144

DOI : $10.4000 /$ pratiques. 2144

ISSN : 2425-2042

Éditeur

Centre de recherche sur les médiations (CREM)

Édition imprimée

Date de publication : 5 juin 2014

Référence électronique

Luc Maisonneuve, "Formes du savoir, sens du savoir », Pratiques [En ligne], 161-162 | 2014, mis en ligne le 05 juin 2014, consulté le 14 novembre 2019. URL : http://journals.openedition.org/pratiques/ 2144 ; DOI : 10.4000/pratiques.2144

Ce document a été généré automatiquement le 14 novembre 2019.

(c) Tous droits réservés 


\section{Formes du savoir, sens du savoir}

L'exemple du Loup et l'Agneau de Jean de La Fontaine en classe de CE1 de l'école primaire française

Shapes of Knowledge, Sense of Knowledge. The Wolf and the Lamb by Jean de

la Fontaine in CE1 Class Session (French Primary School)

\section{Luc Maisonneuve}

1 Le travail présenté ici s'inscrit dans une réflexion menée, d'une part, dans le séminaire Action du professeur ${ }^{1}$ (IUFM/ESPÉ de Bretagne - séminaire dirigé par Gérard Sensevy) et l'ouvrage intitulé Le sens du savoir (Sensevy, 2011) et, d'autre part, dans le Groupe ESPÉ de recherche et de production de ressources pour l'éducation et la formation (GERPREF) «Fables et anticipation ${ }^{2}$ » (IUFM/ESPÉ de Bretagne). Cette réflexion met en évidence la place centrale occupée par les savoirs dans toute séance et séquence d'enseignement/apprentissage. Or, ces savoirs ne sont jamais des «ectoplasmes », des entités évanescentes : ils ont toujours une forme, que celle-ci soit manifeste ou qu'elle soit latente. En effet, sans cette forme, une forme à tout le moins, il serait tout simplement impossible d'identifier un quelconque savoir (Billeter, 2012). Ainsi, la relation qui unit savoir et forme peut être comparable à celle qui unit, dans le signe, signifié et signifiant. Je propose donc dans cette courte introduction de considérer la première hypothèse suivante comme point de départ : il n'y a pas et il ne peut pas y avoir de savoir(s) sans forme(s) associée(s) et réciproquement.

2 L'ensemble du travail présenté ci-après a pour objectif d'essayer d'unifier les éléments observés dans les classes via cette relation constitutive d'un (de) savoir(s) et d'une (de) forme(s). Afin de rendre compte de cette relation réciproque (savoir $\rightarrow$ forme et forme $\rightarrow$ savoir), j'émets une seconde hypothèse : toute manifestation de savoir est le résultat d'au moins une sémiose. Il faut alors comprendre le terme sémiose comme le mouvement conjoint au moins d'un savoir et d'une forme. Toute sémiose est ainsi le processus sémiologique qui institue toute manifestation (tout signe? J'y reviendrai) en tant qu'ensemble inséparable d'un savoir (au moins) et d'une forme (au moins). Par conséquent, tout élément identifié et observé - ici dans le cadre scolaire de séances et/ ou de séquences d'enseignement/apprentissage - est le fruit d'au moins une sémiose. 
Penser l'élaboration des observables à travers ce processus sémiologique c'est aussi repenser toute activité, en l'occurrence de l'élève, comme le résultat d'une somme plus ou moins organisée d'activités sémiologiques - sans préjuger pour le moment d'une quelconque hiérarchisation de celles-ci. Les analyses proposées relèvent davantage, nous le verrons, de la sémiotique que de la sémiologie puisque toujours en rapport avec l'étude d'une fable de La Fontaine ${ }^{3}$. La présentation qui suit n'est qu'une ébauche encore maladroite, et peut-être erronée, d'élaboration d'un cadre commun d'analyse de l'activité reposant sur une conception sémiologique de celle-ci.

Les exemples analysés sont empruntés aux travaux du GERPREF «Fables et anticipation » et à deux mémoires de master $\operatorname{EAD}^{4}\left(1^{\text {re }}\right.$ et $2^{\mathrm{e}}$ années) réalisés par Valérie Le Hir, professeure des écoles et membre du GERPREF « Fables et anticipation ». Ils ont comme supports principaux les séances d'étude du Loup et l'Agneau de J. de La Fontaine effectuées dans la classe de CE1 de Valérie Le Hir ${ }^{5}$. Le dispositif dit « d'anticipation » ne sera pas abordé ici.

4 Dans un premier temps, je vais revenir sur la conception sémiologique de l'activité et sur la définition de la sémiose comme manifestation de cette dernière à travers l'analyse des relations entre savoir(s) et connaissance(s), l'un et l'autre termes seront définis. Ceci sera suivi, dans un second temps, de l'analyse d'exemples pris, d'une part, dans le travail du GERPREF «Fables et anticipation » (réflexion, préparation et analyse de séances de classe autour de l'étude de fables de La Fontaine) et, d'autre part, de l'étude effective du Loup et l'Agneau dans une classe de CE1. Cette analyse identifiera un certain nombre de sémioses en jeu lors d'une séance de lecture en classe et montrera combien cette imbrication est source de difficultés pour les élèves. Le cadre de l'analyse sera essentiellement sémiotique. Son élargissement sémiologique ne sera envisagé que de façon marginale. La conclusion reviendra sur l'ensemble des paramètres et, compte tenu de la remarque précédente, insistera sur les éventuelles confusions par les élèves entre les différents niveaux et types de sémioses à effectuer.

\section{Conception sémiologique et relations savoir(s)/ connaissance(s)}

5 Par formes du savoir, j'entends ici les diverses manifestations (paraphrases, reformulations, désignations, institutionnalisations, présentations...) explicites ou implicites du savoir tel qu'il est identifié et tel qu'il circule et s'élabore lors d'une séquence d'enseignement/apprentissage. Ces formes constituent, à strictement parler, le savoir manifesté et par conséquent susceptible d'être reconnu. Lorsque j'écris " savoir manifesté ", il ne s'agit pas de comprendre « manifesté » comme un synonyme d'explicité. L'implicite est aussi une modalité de la manifestation du savoir, autrement dit une manifestation de sa publication. Toute circulation de savoir emprunte ou élabore un langage qui n'est, ou plutôt ne peut qu'être, que la manifestation explicite ou implicite de cette circulation. C'est le propre de tout langage de ne pouvoir échapper à cette contrainte. Il n'est pas ici question pour le moment de codage. Je parle de/du langage et non d'une/de/de la langue. Un symptôme, par exemple, est ainsi déjà un/du langage. Il est manifestation, et ne peut être que cela, d'un/de savoir, même inaccessible. Sans savoir, donc sans «Réel» présumé, il n'y aurait pas même de symptôme, et donc aucune « Réalité » envisageable (Soler, 20096). 
6 Mais l'usage que j'ai fait jusqu'ici du terme «savoir » doit être maintenant précisé. En effet, je l'ai utilisé comme terme générique, valant à la fois pour savoir et connaissance, en un sens assez proche du knowledge anglo-saxon qui ne différencie pas les deux acceptions. Or, faire une distinction entre savoir et connaissance me semble essentiel et je vais m'en expliquer plus bas. Il me faut néanmoins résoudre auparavant le problème lexicologique suivant: le mot "savoir" sert à la fois à désigner le savoir comme " tout ", c'est-à-dire en tant que "savoir et connaissance ", le knowledge anglo-saxon c'est l'utilisation que j'en ai faite depuis le début de cet article - et le savoir comme «partie» de ce «tout» dans l'expression «savoir et connaissance». Je propose, au moins le temps de cet article et à partir de maintenant, de nommer savoir, en police italique, le savoir en tant que "tout» et de conserver le mot "savoir", en police romaine pour l'une des deux parties de ce "tout», autrement dit : savoir = savoir + connaissance.

7 Tout savoir, en tant que Réalité, relève ainsi d'une construction sociale et culturelle. Il n'y a pas de savoir hors le monde, hors une communauté d'êtres-au-monde. En cela, il appartient à et vit dans une institution (Douglas, 1986-2004). L'institution est très exactement le devenir-Réalité et donc, en ce sens, un devenir atemporel, décontextualisé et dépersonnalisé : abstraction totale (produite) et conséquemment intégralement concrète (avérée). Le savoir est alors le bien de tous et n'est celui de personne. Le langage, un langage, en est toujours sa manifestation ${ }^{7}$. Sans que ce langage ne soit nécessairement codé, il s'inscrit dans un devenir-code. Toute institution produit en effet de la formulation et donc de la formalisation. "Il n'y a pas de savoir sans une pratique discursive définie; et toute pratique discursive peut se définir par le savoir qu'elle forme » (Foucault, 1969:238-239). Cette production est une tension continue à la reproduction, au maintien "en l'état [du] monde familier dans lequel nous vivons " (Billeter, 2012 : 34). Institutionnaliser, c'est se donner un rapport au monde stable, au moins pour un temps déterminé. «Si je veux qu'une porte tourne, il faut que les gonds soient fixes»(Wittgenstein, 1965: § 343). Il nous faut donc pour cela créer de la mémoire collective, stocker des données. L'institution est l'ensemble de ces données organisées, hiérarchisées, régulées - construites autour d'une communauté d'intérêts et par une communauté d'intérêts. La formalisation est le réquisit incontournable de tout de "vivre ensemble » et comme toute formalisation est une formalisation de savoir(s), celui(ceux)-ci est(sont) en quelque sorte incarné(s) dans et par l'institution. "Voici l'enfance des sociétés : se doter d'institutions qui leur permettent de maintenir les croyances de leurs habitants en état, malgré les démentis de la réalité [ici, réalité = Réel]. Et pour que ces institutions survivent, il leur faut des récits » (Lambert, 2013). Ces récits pourraient être décrits comme l'ensemble des savoirs et connaissances disponibles à un moment donné, quelque chose comme l'encyclopédie d'Eco (1985). Or, ces récits requièrent un apprentissage et donc une médiation. En effet, pour être transmis et appris, pour être mémorisé, le savoir doit faire l'objet d'une contextualisation (temps, espace, sémiotisation). Cette contextualisation n'est que la prise en compte du constat que jamais nous n'apprendrons le savoir, mais juste des savoirs et des connaissances situés. Si, comme l'écrit G. Sensevy (2011:60), « un savoir [...] est une puissance d'agir [...] en situation ", cette puissance d'agir en situation est très précisément ce que j'appelle connaissance (voir aussi Margolinas \& Laparra, 2011 : 20-21). Le double processus de contextualisation/décontextualisation rend compte du constant aller-retour des savoirs aux connaissances et de celles-ci aux premiers. J'appelle alors « expérience » aussi bien l'institutionnalisation (ou décontextualisation), 
c'est-à-dire le passage des connaissances aux savoirs, que la dévolution (ou contextualisation), c'est-à-dire l'inverse. Autrement dit, tout savoir est issu d'une connaissance et toute connaissance ne s'inscrit que dans un savoir, l'un et l'autre requérant une forme, un signifiant. Institutionnaliser, c'est donner forme au couple savoir-connaissance, forme qui ne peut être considérée qu'en tant qu'«assertion garantie » selon les termes de Dewey (2006), c'est-à-dire en tant qu'assertion valide à ce moment-là.

éves, comme tout apprenti, manipulent et, subséquemment, apprennent, dans un premier temps, des connaissances. Celles-ci restent très largement implicites dans la mesure où elles relèvent de leur usage en situation. Elles ne deviennent des savoirs qu'après explicitation, décontextualisation, secondarisation (Sensevy, $2011: 160-161)^{8}$. Dans le couple savoir-connaissance, il y a donc un changement de forme : d'une forme en acte de la connaissance à une forme institutionnalisée du savoir, d'une forme par conséquent à dominante idiosyncrasique à une forme à dominante publique.

Il y aurait donc deux processus conjoints : l'un qui consisterait à installer en situation des connaissances (la dévolution de la théorie de l'action conjointe en didactique désormais TACD -, Sensevy \& Mercier, 2007) et le second à transformer ces dernières en savoirs, ou institutionnalisation, selon les termes de la TACD. Ces deux processus vont de pair. Ils sont en relation logique et temporelle l'un avec l'autre. En effet, il est impossible d'institutionnaliser hors contextualisation initiale car il n'y aurait rien à institutionnaliser'. Ceci a pour conséquence immédiate de s'interroger sur ce qui va guider l'ensemble dévolution/institutionnalisation. Autrement dit : comment les élèves se repèrent-ils dans cet ensemble ? Á quels signes, à quels faits saillants, doivent-ils être attentifs ? Comme l'écrit B. Schneuwly (2000: 23), nous sommes en présence d'une double sémiotisation :

«Un objet d'enseignement est toujours et nécessairement dédoublé dans la situation didactique: il est là, rendu présent, "présentifié", par des techniques d'enseignement, matérialisé sous des formes diverses (objets, textes, feuilles, exercices, etc.) en tant qu'objet à apprendre, à "sémiotiser" [...], à propos duquel de nouvelles significations peuvent et doivent être élaborées par les élèves; et il est là en tant qu'objet sur lequel celui qui a l'intention d'enseigner guide l'attention de l'apprenant par des procédés sémiotiques divers, sur lequel l'enseignant pointe ou montre des dimensions essentielles en en faisant un objet d'étude, ce guidage entrant d'ailleurs dans la construction même de l'apprentissage. Les deux processus - rendre présent l'objet et le pointer / montrer - sont indissolublement liés, se définissant mutuellement. »

Sensevy (2011 : 72) montre que cette sémiotisation relève d'une "sémiose d'autrui dans le savoir » ou plutôt d'" une sémiose du savoir à travers autrui » et qu'elle ne peut advenir sans ajustements constants (ibid. : 122). En effet, tout le processus repose, d'une part, sur le guidage enseignant, autrement dit sur son degré de réticence et de sémiotisation du savoir en jeu, et, d'autre part, sur la capacité des élèves à vaincre cette réticence (apprendre le savoir nouveau) en s'appropriant, décodant, construisant, le(s) système(s) sémiotique(s) adéquat(s). La réticence part du constat que tout enseignement a une part d'inconnu et qu'il n'est pas possible de connaitre celui-ci avant d'en avoir fait l'apprentissage, de l'avoir transformé en connu. La réticence est donc la part d'inconnu de toute situation d'apprentissage, ce que l'enseignant ne dit pas ou pas tout de suite. Elle a pour corollaire ce que Sensevy (2008) appelle la «clause proprio motu» (de son propre mouvement) ou capacité à s'emparer seul de l'apprentissage, à en prendre la responsabilité. Cette capacité à vaincre la réticence met 
en jeu des connaissances. À ce titre, toute sémiose est une expérience. Elle est même l'expérience de la Réalité et la constitution de celle-ci. Les connaissances en sont l'une des modalités pratiques d'exécution: elles sont requises et elles s'élaborent dans le temps de la situation d'apprentissage. Elles n'ont donc pas nécessairement besoin d'être immédiatement reconnues. Elles sont en revanche indispensables pour la réalisation de la tâche dans la situation considérée, d'où un contrôle magistral toujours délicat de la réticence. Gombert (1990) parle en l'occurrence de connaissances dites épi qu'il oppose aux connaissances dites méta (les savoirs selon ma terminologie) :

«L'explication de procédures, démarche souvent utilisée par le pédagogue, ne peut être profitable pour les apprentissages que si l'enfant est capable d'une gestion consciente de ses tentatives de résolution. Elle sera inutile si elle porte sur des connaissances, qui, n'ayant pas encore atteint un niveau de maitrise épicognitive stabilisée, ne peuvent en aucun cas accéder à un statut métacognitif. Dans un tel cas, l'action pédagogique doit viser à la constitution et à la stabilisation de connaissances épicognitives correspondantes, ce qui passe essentiellement par la manipulation d'objets (linguistiques pour ce qui nous intéresse) [expérience, connaissances, dévolution] dont les règles de fonctionnement correspondent aux règles qu'il s'agira ultérieurement de faire maitriser à un niveau conscient [en tant que savoir(s), institutionnalisation]. C'est par des manipulations que l'enfant sera conduit à la maitrise fonctionnelle indispensable à l'efficacité des efforts qui ultérieurement viseront à la compréhension. » (ibid. : 253).

11 Apprendre, c'est donc faire d'incessants allers-retours entre les connaissances et les savoirs. Toute institutionnalisation partielle oblige à reconsidérer et reconfigurer la dévolution en cours, ce qui entraine de nouvelles institutionnalisations et ainsi de suite. Dans les classes, et je vais y revenir plus bas, ces institutionnalisations successives portent souvent le nom de "validations » : l'enseignant valide ou invalide une réponse, un comportement. Les élèves doivent donc être très attentifs pour saisir et s'approprier ces « validations » sous peine de ne pouvoir réinterpréter et, si besoin, réorienter leur expérience. Or, pour les faire siennes, pour en faire son miel, il ne leur suffit pas d'être attentifs, il leur faut aussi en comprendre le bien fondé. Ils sont alors confrontés à la réticence enseignante ci-dessus mentionnée : trop de réticence, ils ne comprennent pas, trop peu, ils n'apprennent pas. Et ceci ne concerne pas les seuls apprentissages "disciplinaires». Chaque expérience est une imbrication de multiples sémioses et chaque situation de classe une imbrication d'expériences. Dans ce qui va suivre, je n'ai pas l'ambition de rendre compte d'une telle complexité. Qui d'ailleurs le pourrait? Je veux juste essayer de mettre en évidence quelques-unes de ces imbrications, de ces expériences et de ces sémioses.

\section{Analyse du Loup et l'Agneau dans une classe de CE1}

Pour réaliser cela, comme je l'ai écrit plus haut, je vais désormais essentiellement me servir du travail effectué par le groupe GERPREF «Fables et anticipation » et plus particulièrement des séances réalisées en classe de CE1 et analysées par Valérie Le Hir. J'aimerais ainsi montrer que le sens du savoir, «le(s)" sens de la fable Le Loup et l'Agneau de J. de La Fontaine, se construit simultanément aux formes qui le(s) manifestent lors des multiples processus de sémiotisation en relation plus ou moins serrée avec le texte de La Fontaine. Le domaine générique sera donc celui de la lecture, voire de la lecture littéraire, et de son enseignement/apprentissage. Par ailleurs, parler de construction simultanément du sens et des formes de celui-ci me situe dans une 
perspective fishienne (Fish, 2007), perspective selon laquelle c'est la lecture d'un texte qui fait le texte. Ceci ne veut pas dire, bien entendu, qu'il n'y a pas de texte matériel, objet du monde, qui préexiste à l'activité des lecteurs : nous lisons toujours quelque chose. Il faut ici entendre que le texte ne sera jamais lu, donc compris et interprété, tel qu'un improbable (un impossible ?) lecteur modèle aurait pu ou dû le lire (Eco, 1985).

Toute lecture est nécessairement située. Autrement dit, elle n'est jamais que le produit d'une communauté et d'un environnement (physique et social). Il ne peut pas y avoir, par conséquent, de lecture vraie ou de lecture ultime. Chaque lecture reprend, pour son compte, tout ou partie des lectures antérieures (Foucault, 1971). Il se constitue ainsi, au fil de celles-ci, un texte lui-même objet de lecture(s), et cela à l'infini. Chaque nouvelle lecture reprend donc cet héritage et inaugure une forme/sens inédite. Dans un premier temps, je vais utiliser le terme de paraphrase pour désigner cette forme/sens. Elle sera alors, selon cette acception, le résultat de la construction sémiotique partagée par la communauté de lecteurs retenue, en l'occurrence une classe de CE1. Le terme de paraphrase et son choix seront précisés lors de l'analyse.

Mais avant toute précision supplémentaire, il me parait important de souligner que l'utilisation du terme paraphrase a pour conséquences de poser ou reposer un certain nombre de questions, par exemple: dans quelle(s) paraphrase(s) sommes-nous en ce moment? Dans ou avec quels ajustements de sens construisons-nous une (des) forme(s) commune(s) de paraphrase ? Quelle(s) institutionnalisation(s) - on line et provisoire(s) de ces ajustements est(sont) produite(s) lors de chacune des situations considérées et en quoi viennent-elles contribuer à une sorte d'ultime " paraphrase des paraphrases »? Quelles sémioses faut-il réaliser pour comprendre les paraphrases intermédiaires et les intégrer dans un tout cohérent et pertinent? Etc. Ces quelques questions montrent combien la paraphrase, selon l'acception proposée ici, pourrait être un synonyme de la Réalité. Chaque paraphrase est une lecture du Réel, une sémiose de celui-ci. Par conséquent, elle rend compte, à sa manière, de ce qu'il peut être, non de ce qu'il est.

Le GERPREF «Fables » a tout autant produit ses formes du/de savoir qu'il en a élaboré un/des sens. Lors de l'étude du Loup et l'Agneau ou Des animaux malades de la peste, les paraphrases des textes de La Fontaine, mais aussi celles des textes de G.-E. Lessing (2008), de N. Cartwright (2006), de B. Daunay (2002a, 2002b) ou de L. Lefeuvre (2008), par exemple, ont toutes contribué à une paraphrase collective tant des fables choisies que des dispositifs didactiques retenus. Celle-ci a alors constitué l'arrière-plan épistémique des séances d'étude en classe. Ce sont ces différentes formes/sens paraphrastiques collectives, leurs déclinaisons, leurs interactions et leurs coconstructions, que je me propose d'examiner ici. Je vais pour cela tenter de décrire et d'analyser quelques-unes des sémioses de savoirs et de connaissances (désormais S/C) repérables dans les diverses préparations des séances (collectives et individuelle), leurs retranscriptions, les divers écrits produits et, enfin, les commentaires et analyses des mémoires de master EAD réalisés par Le Hir (2012).

Pour parvenir ne serait-ce qu'à une description succincte de ces sémioses, il m'a fallu identifier ce qui pouvait (pourrait?) être considéré comme «signe» de $S / C$, voire comme « faisant signe » de S/C. Or, cette identification est extrêmement problématique dans la mesure où le signe, ou plutôt le signe de..., est très précisément, et presque de manière tautologique, ce qui fait signe : autrement dit le signe se signale en tant que signe et, conséquemment, ne peut se signaler qu'en tant que signe de quelque chose, ici de $\mathrm{S} / \mathrm{C}$. Il fallait donc me résoudre à ne pouvoir chercher que ce que je présumais être 
là : une fable, une classe, un collectif... Il devait y avoir explicitement ou implicitement des marques de ces éléments. J'ai alors cherché non les signes de $\mathrm{S} / \mathrm{C}$ mais, d'une part, les formes ou manifestations de $\mathrm{S} / \mathrm{C}$ (les signes) et, d'autre part, la formation ou processus d'émergence de ces derniers (sémiose).

Je suis parvenu à identifier les cinq observables suivants :

- 2.1. lexique et métalangage ;

- 2.2. matériel, support, organisation de la classe ;

- 2.3. habitus, intertextualité, explicite et implicite textuels ;

- 2.4. tâches et activités ;

- 2.5. ajustements, savoirs/connaissances du professeur et des élèves.

18 Cette liste n'a pas la prétention d'être exhaustive. Son examen me permettra de revenir en conclusion sur les notions de paraphrase et de désignation, comme sur celles connexes de sémiose et de sémiotisation.

\subsection{Lexique et métalangage}

19 Un certain nombre de termes métalangagiers apparaissent tout au long du processus didactique : personnage, phrase, paroles, dialogue, « un p'tit trait avant » (le tiret des dialogues), recueil (de fables), point, commentaire, justification («Ben, vu qu'il en a déjà dit trois, certainement là encore, c'est une nouvelle accusation qui n'est pas justifiée ", S4TDP $195^{10}$ ), écrivain, narrateur... Ces termes semblent faire partie de la classe. Bien que relevant du commentaire, ils sont si intimement mêlés aux diverses paraphrases du texte qu'ils semblent le plus souvent en être une composante indissociable. Seuls ceux qui sont reformulés (le tiret, par exemple) sont nouveaux et acquièrent, au moins provisoirement, le statut d'outils commentatifs. Si tous ces termes sont, pour l'enseignant et l'observateur, le signe d'un savoir littéraire permettant d'identifier des éléments du texte à lire, il est permis de s'interroger sur leur statut pour les élèves. En effet ces derniers sont censés les décoder et les interpréter sans ambiguïté, c'est-à-dire les interpréter comme outils servant une fin et non comme une fin en soi. Il y a, semble-t-il, une confusion entre utiliser ce qu'on pourrait appeler une boite à outils littéraire et l'objet de cette utilisation. Ainsi le terme « personnage » est-il pour les élèves un terme métalangagier ou une paraphrase du texte? Je reviens plus bas sur cette difficulté.

Si l'on peut s'interroger sur le statut des termes métalangagiers, il n'en est pas de même pour un certain nombre de mots ou expressions de la fable, par exemple : l'an passé, médire, point dans " ne... point...", se venger, aimer les agneaux... la liste est longue. C'est autant la langue du XVII ${ }^{e}$ siècle qui est expliquée que les situations qu'elle recouvre. "Médire» va ainsi faire l'objet d'un long développement qui portera sur la fable de La Fontaine et sur des exemples actualisés (Le Hir, 2012: 50). Or, si l'on suit F. Rastier (2003), il ne peut y avoir de sens sans signification et, corrélativement, la signification, en tant que signe isolé, n'a pas de sens. La sémantique relève toujours d'une contextualisation :

«Convenons que la signification est une propriété assignée aux signes, et le sens une "propriété" des textes. La notion transitoire de contexte peut servir à opposer ces deux problématiques. Si l'on approfondit la distinction entre sens et signification, un signe, du moins quand il est isolé, n'a pas de sens, et un texte n'a pas de signification. La signification résulte en effet d'un processus de décontextualisation, comme on le voit en sémantique lexicale et en terminologie: d'où son enjeu 
ontologique, puisque traditionnellement on caractérise l'Être par son identité à soi. En revanche, le sens suppose une contextualisation maximale aussi bien par la langue (le contexte, c'est tout le texte) que par la situation (qui se définit par une histoire et une culture, au-delà du hic et nunc seul considéré par la pragmatique). Aussi, alors que la signification est traditionnellement présentée comme une relation, le sens peut être représenté comme un parcours [c'est l'auteur qui souligne]. »

21 C'est donc par la contextualisation, c'est-à-dire par l'expérience, que s'élaborent la description et l'explication. Il n'y a pas de terme qui ne soit ainsi référencé à une ou plusieurs situations, à un ou plusieurs agencements (Deleuze, 1996). Pour notre exemple : implicitement en ce qui concerne les termes métalangagiers, explicitement pour le vocabulaire de la fable.

Ce premier ensemble d'observables montre que les élèves doivent effectuer des sémioses croisées entre, d'une part, le texte de la fable et, d'autre part, les "outils littéraires " utilisés. Pour que cette double sémiose fonctionne, il faut que les deux sous-ensembles considérés (1.texte de la fable, 2. outils littéraires) soient en adéquation et que simultanément, la question mérite d'être soulevée, la pertinence des choix magistraux successifs quant à leur sélection soit assurée. À moins de considérer que deux objectifs principaux soient envisagés lors de cette séance (1. lire et comprendre Le Loup et l'Agneau, 2. apprendre à lire et à comprendre un texte littéraire), ce que ne mentionne pas la préparation, il est en effet permis de s'interroger sur l'apport de tel ou tel outil littéraire pour la compréhension du texte par des élèves de CE1. Bien au contraire, le recours au commentaire implicite, via les termes métalangagiers, est loin de faciliter la compréhension du texte pour de nombreux élèves qui ne maitrisent pas ou mal ces termes et leur rapport au texte de la fable.

\subsection{Matériel, support, organisation de la classe}

23 Le matériel et les supports utilisés sont autant de signes des S/C en jeu : crayons de couleur, surligneurs (dialogue : "ce qu'on a mis en bleu »), feuilles, tableaux (tableau mural, tableau d'évaluation qui porte sur l'identification des locuteurs - reprises anaphoriques, tableau TPC (pour texte, paraphrase, commentaire), étiquettes (pour écrire), texte de la fable. Le tableau TPC a été élaboré pour rendre compte de la difficulté rencontrée à différencier la paraphrase et le commentaire. C'est lors des échanges à propos du Loup et l'Agneau, dans le GERPREF « Fables et anticipation ", que cette difficulté a tout d'abord surgit: "C'est au cours d'une séance de travail du collectif de recherche [le GERPREF] autour de la signification du récit de la fable Le Loup et l'Agneau que la paraphrase est apparue comme un moyen incontournable au service de la compréhension-interprétation du texte. En élaborant une paraphrase collective, les membres du groupe se sont aperçus de deux choses. D'une part, les mots du texte ne renfermaient pas les mêmes significations pour tous. D'autre part, deux types de paraphrases se mélangeaient: une paraphrase-traduction et une paraphrasecommentaire » (Le Hir, 2013 : 16). La paraphrase, comme le fait remarquer B. Daunay (2002b : 133), postule qu'il « est possible de reformuler le sens [...], ou le même sens [...] » mais, précise-t-il, « la question de l'identité sémantique n'est pas réellement posée : le fait qu'il soit possible de dire la même chose en d'autres termes n'est jamais problématisé ». Le tableau TPC, en explicitant les différences entre texte, paraphrase et commentaire, permet une première problématisation : les élèves doivent s'interroger 
sur ce qui relève de l'une ou l'autre des trois catégories du tableau. Par ailleurs, chaque élément, et par conséquent chaque catégorie, est le résultat d'une sémiose ou plutôt d'un emboitement de sémioses. Par exemple : il n'y a pas de surligneurs sans quelque chose à surligner. Le surligneur est déjà, avant le surlignement et donc avant l'élément surligné, une première sémiose. Les élèves savent en effet qu'ils n'auront pas à surligner tout le texte mais seulement certaines de ses parties qui seront alors, de fait, signalées comme parties remarquables, autrement dits comme éléments de $\mathrm{S} / \mathrm{C}$ importants. De la même façon, remplir tel ou tel tableau revient à accepter les catégories mêmes qui président à leur organisation et au fonctionnement tabulaire. Ces catégories servent de système(s) organisateur(s) de ce qui est à construire (Goody, 1979). On retrouve, mais d'une autre manière et sous une autre manifestation, le métalangage déjà relevé. Nous sommes à tout le moins en présence d'une triple sémiotisation plus ou moins explicite : celle qui signale le tableau (implicite : on ne dit pas ceci est un tableau), celle qui signale les catégories du tableau (explicite : les entrées du tableau, explicites mais difficiles car relevant du métalangage), celle qui signale la tâche à effectuer (explicite ou implicite: remplir le tableau, c'est-à-dire mettre en relation des catégories et des contenus textuels).

Tout ceci contraint l'activité de lecture, tout comme vont la contraindre les dispositifs de travail en groupe ou l'utilisation d'un rétroprojecteur. Chaque fois, la sémiose attendue prédispose une façon de faire. La reconnaissance simultanée (par l'ensemble des acteurs) des signes de la situation didactique constitue la relation comme relation contrat-milieu didactique selon le dispositif matériel et l'organisation de la classe retenus. Les contraintes liées à ces choix (milieu) sont autant de contrats didactiques à respecter. Tout changement de formes est un changement de contenu de S/C.

\subsection{Habitus, intertextualité, explicite et implicite textuels}

Par habitus, j'entends ici tout ce qui dans le texte de la fable va éveiller chez le lecteur des scénarios potentiels issus de souvenirs. Ainsi, par exemple, le mot "rivière " éveillera toutes les scènes de rivière déjà rencontrées et tous les récits personnels (ou intertextuels, voir ci-dessous) liés à celles-ci. Il en sera de même avec les mots "frère ", « chiens » ou « bergers ». L'anthropomorphisme rappellera telle ou telle histoire, tel ou tel jeu. Les élèves font ainsi constamment référence à leurs souvenirs personnels : « Ya mon papy qui... » (Le Hir, 2012, S4, TDP225), « sa famille l'a insulté » (ibid., travail en groupe, TDP3, annexe 13, p. 114), réflexion sur la justice et le droit : «parce qu'il a pas droit! / Ouais... il a pas droit sans ... sans autorisation / Ben quelle autorisation? / Ben... faire un combat et pour qu'il gagne... / ... / c'est pas juste » (ibid., S4, TDP398-404, annexe 14, p.130)... Tout ceci relève d'expériences plus ou moins partagées par l'ensemble des élèves et présentes dans le texte de La Fontaine (à tout le moins par l'intermédiaire de ses personnages). Pour expliquer les difficultés du texte, P (pour professeure des écoles) ne cherche pas à rendre compte d'une éventuelle signification " historique» de celui-ci, mais se saisit des acceptions et/ou des situations actuelles pour y revenir. Par exemple: le bord de la rivière dans la fable devient un bord de rivière "connu » des élèves... L'appel à l'habitus des élèves peut être vu comme une sémiose par actualisation des sèmes du texte (Citton, 2007) et recours aux « transactions personnelles » (Terwagne \& Vanesse, $2008: 74$ sqq.). 
L'intertextualité entre en relation avec l'habitus. Ainsi des trois principaux réseaux intertextuels du texte de La Fontaine: celui de son origine (par exemple: le texte d'Ésope ou celui de Phèdre), celui du thème (par exemple: Le Petit Chaperon rouge: le loup mange le chaperon rouge; Le Loup et les Sept Chevreaux : le mensonge, la mauvaise foi) et celui du genre textuel (par exemple : La Cigale et la Fourmi (la fable). La sémiose se fait essentiellement ici par comparaison : c'est comme dans... cela nous rappelle telle situation... Le fonctionnement de cette sémiose est très proche de la précédente. Mais alors que l'habitus faisait plutôt référence aux scénarios de l'environnement quotidien des élèves, l'intertextualité fait plutôt référence aux scénarios de l'environnement culturel de ceux-ci («transactions intertextuelles», ibid.). Nous sommes à chaque fois très proches de ce que G. Brousseau appelle un contrat d'ostension (Brousseau, 1998 ; Sensevy, 2011), contrat qui semble fonctionner ici selon le principe qu'il est suffisant de montrer le lien - de le dire en l'occurrence - pour que celui-ci, alors devenu «signe de... ", produise une connaissance sur ce dont il est le signe, mais signe de quoi ? Ainsi, par exemple : de quoi Ésope, Le Petit Chaperon rouge, les rimes, sont-ils le signe? Quel est, quels sont, le(s) S/D(s) visé(s) ? Que doivent faire les élèves ?

Le recours au texte explicite (ou explicite textuel) permet cette ostension - P montre, désigne, un mot, une expression - et confère par ce fait le statut de signe à l'élément montré. La sémiose opère donc dans ce cas par ostension "directe ", mais aussi, cas le plus fréquent, sur ou à partir de l'implicite du texte (ou implicite textuel - relevant des présupposés), cette fois sous formes de paraphrases du texte original (on pourrait alors parler d'ostension «indirecte »). Ces paraphrases relèvent davantage d'une sorte de commentaire paraphrastique de la fable. J'emploie l'expression «commentaire paraphrastique" dans la mesure où il est difficile, dans ces interventions, de différencier le commentaire de la paraphrase, comme il est difficile de faire la part, dans ces commentaires et/ou paraphrase de ce qui relève de l'explication ou de l'interprétation. Lorsqu'un membre du GERPREF, par exemple, parle de "surjustification du loup » dans les dernières paroles de celui-ci ${ }^{11}$, que fait-il exactement ? Il paraphrase en résumant l'ensemble des propos? Il commente en prenant position sur ces derniers? Ce faisant, il les évalue ainsi que le comportement du loup. Il les interprète et les explique en même temps. Il explicite aussi sa propre compréhension/ interprétation de la fable et par la même occasion, il s'interroge sur ce besoin de justification du loup, d'où le "sur » de "sur-justification », dans la mesure où, de toute façon, le loup mange l'agneau dans les vers suivants. La question posée alors par le GERPREF aurait pu être celle de la possibilité ou plutôt de l'impossibilité d'un commentaire non paraphrastique. Non qu'il ne faille différencier paraphrase et commentaire, cela est peut-être pertinent, je ne vais pas ici en débattre, mais dans l'intention de s'interroger sur cet horizon de toute lecture où se profilerait toujours un autre texte, plus originel, plus vrai, que le commentaire ferait alors advenir et que le texte aurait caché jusque-là (Foucault, 1971).

\subsection{Tâches et activités}

Les tâches demandées aux élèves (surligner, souligner, colorier, expliquer, répondre à des questions, s'écouter, prendre en compte les réponses des autres, faire des allersretours entre les scénarios communs mobilisés et la fable étudiée, recourir à des scénarios intertextuels, travailler en groupes, paraphraser le texte, récupérer les amorçages de $\mathrm{P}$, expliquer...) sont toutes, peu ou prou, des procès d'ostension 
«indirecte». P, ne pouvant dire ce qu'il faut trouver (réticence - Sensevy, 2011), installe un chemin pour y parvenir. La difficulté tient au fait que $\mathrm{P}$ est seule à déjà connaitre ce chemin et son aboutissement. Les élèves doivent donc effectuer quelque chose comme une série de sémioses progressives orientées par $\mathrm{P}$ au fur et à mesure de l'avancée didactique sans pouvoir à aucun moment, sauf au terme du parcours, se représenter la cohérence globale de celles-ci. Ainsi, par exemple, de l'utilisation du dialogue entre le loup et l'agneau : tâche 1 : matérialiser les éléments du dialogue entre le loup et l'agneau (surlignage et coloriage); tâche $2 a$ : paraphraser le texte de La Fontaine; tâche $2 b$, "traduire " la langue de La Fontaine (la différence entre ce qui relève de la paraphrase de "traduction » littérale et ce qui relève de la paraphrasecommentaire - utilisation de l'outil TPC; tâche 3 : mettre en valeur des mots du texte qui serviront de points d'appui lors de la restitution. Chaque étape est le résultat d'une sémiose intermédiaire devant conduire, par intégrations successives, à la compréhension du dialogue lui-même partie de la compréhension globale de la fable. Chacune de ces sémioses est présupposée pertinente en regard de l'objectif final, mais aussi dans ses relations avec les sémioses précédentes et suivantes. Nous retrouvons ici la pertinence des différents choix didactiques effectués. Autrement dit, $\mathrm{P}$ présuppose une forme de progressivité sémiotique reposant sur des sémioses de type spiralaire. Les institutionnalisations successives ont ainsi pour finalité de garantir le bon déroulement de ces sémioses et leur intégration à la sémiose globale du texte. Malgré tout, je le souligne encore, aucun élève n'est en mesure d'évaluer la pertinence et la cohérence de ces institutionnalisations avant le terme de la séance.

Les élèves ne peuvent donc prendre la responsabilité de leurs apprentissages (clause proprio motu) que lors des sémioses intermédiaires. Ils ne construisent donc, au mieux, que des éléments de compréhension locaux. La sémiose de ces éléments ou synthèse globale du sens reste à faire. Elle ne peut de toute manière advenir qu'au terme de l'étude par une ultime reprise paraphrastique et/ou un commentaire. Les élèves doivent donc accepter ce contrat. Il ne dépend pas d'eux de comprendre trop vite ou de comprendre autrement. Le guidage, ou gestion de la réticence, est très difficile à contrôler. Le choix d'un dispositif didactique global de type " cours dialogué " permet à la fois de conserver le contrôle magistral - les validations ou invalidations successives en sont le témoignage - et d'en masquer le caractère magistral (Bonnery, 2007). Ce dispositif appelle donc à effectuer de très nombreuses sémioses en ligne, au fil des TDP, sans que leur bien-fondé soit toujours facile à identifier. Les processus de validation sont souvent implicites : un regard, la position du corps, l'appel à tel ou tel, une remarque générale, une trace au tableau, etc. Tout cela concourt à brouiller tâches et activités et font basculer constamment le dispositif sémiotique déjà extrêmement complexe vers un dispositif sémiologique qui l'est encore davantage. Et :

" comme l'enseignant institutionnalise la progression de la lecture du texte lors des "corrections" de ces tâches, l'élève ne peut mesurer, implicitement, la qualité de sa lecture qu'à l'aune de la réalisation de ces tâches. Celles-ci se substituent ainsi doublement à la lecture et à son apprentissage. Les élèves apprennent ainsi davantage à répondre à des questions qu'à lire ou apprendre à lire, voire même, pour les élèves en difficulté, à se donner l'illusion qu'ils répondent alors, qu'à proprement parler, ils ne font qu'attendre des réponses » (Maisonneuve, $2010: 167-168$ ). 


\subsection{Ajustements, savoirs/connaissances du professeur et des élèves}

parlerai ici «que» des ajustements didactiques en jeu: par exemple, ajustements élève(s)-élève(s), élève(s)-P, élève(s)-P-S/C, élève(s)-P-fable, élève(s)-Pparaphrases du texte... contrôle de $\mathrm{P}$ sur le temps didactique, présence de plus en plus grande de $\mathrm{P}$ en fin de séance, etc.

relation, chaque transaction, est un ajustement conjoint plus ou moins réussi et plus ou moins repéré par les différents protagonistes. Ainsi de la morale de la fable: $\mathrm{P}$ reconnait difficile d'imaginer s'il est possible de deviner (de trouver ?) la morale sans l'avoir déjà lue. $\mathrm{P}$ est en effet, comme je l'ai écrit plus haut, en relecture lors des séances de classe. Elle sait pourquoi elle demande et institutionnalise ceci ou cela, et, puisqu'elle sait cela, tout fait signe(s) selon son projet (son intentionnalité). Le dispositif didactique pensé en amont a été pensé comme un ajustement présumé, principalement, d'une part, aux réquisits du texte, d'autre part, aux $\mathrm{S} / \mathrm{C}$ des élèves (affordances texte/élèves/projet magistral - Gibson, 1986 ${ }^{12}$ ). Les ajustements reposent sur les capacités de sémioses conjointes des divers protagonistes de la situation, sur leurs capacités à s'ajuster " on line » à partir de ces sémioses, autrement dit à ne pas se méprendre ni sur les diverses intentionnalités, ni sur les S/C en jeu (Sensevy, 2011 : 160-161). De nombreux travaux ont montré combien des échecs répétés à ces ajustements, notamment la méprise quant aux sémioses attendues, peuvent être sources de difficultés scolaires (Lahire, 1993; Peltier, 2004 ; Rocheix \& Crinon, 2011). Lors des séances de classe observées, Le Hir (2013 : 95) fait le commentaire suivant, à la suite des réponses des élèves quant aux troisième et quatrième mensonges du loup :

«Les deux premiers mensonges sont parfaitement invalidés par les faits sur lesquels s'appuie l'agneau. Il ose d'ailleurs l'exprimer avec de longues phrases révérencieuses. Le troisième mensonge également contredit par l'agneau, l'est d'une manière beaucoup plus laconique ("Je n'en ai point"). Quant au dernier, il n'y a pas de défense de l'agneau qui a compris que son heure était venue. Ce déséquilibre entre les arguments du loup et ceux de l'agneau a peut-être été interprété par les élèves comme une preuve (même injuste) du droit du loup de manger l'agneau. Car, enfin, la fin de cette fable étant totalement immorale, les élèves préfèrent peut-être croire que l'agneau est quand même un peu responsable de ce dont l'accuse le loup. C'est ainsi plus supportable émotionnellement. »

Peut-être... L'ajustement n'est pas facile : il faut conjecturer. Par ailleurs, Le Hir (ibid.) indique que dans une autre classe, l'accent ayant été fortement mis sur cet aspect de l'argumentation, les élèves répondent différemment. Nous retrouvons ici la question du contrôle de la réticence. Le guidage fort de la seconde classe évite en effet les incertitudes, mais c'est peut-être au détriment d'une appropriation plus personnelle de la fable. Il est impossible de trancher comme il est impossible de tout contrôler, de tout tenir ensemble : tout gain appelle une perte et s'ajuster, c'est toujours se tenir sur un fil. Dans une classe, nul n'est ainsi jamais certain de ces ajustements. Il faut souvent faire « comme si... », faire des paris. La redondance et la répétition didactiques sont les traces de ces ajustements permanents. 


\section{Conclusion : paraphrase, désignation, sémiose, sémiotisation} texte lors de la première lecture de celui-ci en vue de l'étudier en classe (passage de l'objet culturel, ici Le loup et l'Agneau, à l'objet à enseigner - première « didactisation ») ; b. paraphrase lors de la préparation des séances d'étude en classe et de l'identification/ anticipation des difficultés présumées des élèves (par exemple: Pourquoi le loup ne mange-t-il pas l'agneau tout de suite ? Pourquoi « ment-il »? Etc.) (passage de l'objet à enseigner à l'objet d'enseignement - Schneuwly \& Dolz, 2009); c. paraphrase encore lors des séances elles-mêmes (passage de l'objet d'enseignement à l'objet enseigné ibid.): usage d'outils sémiotiques divers (résumés, commentaires du texte, renseignements de tableaux, par exemple le tableau TPC, etc.), ajustements, institutionnalisations, etc. Les différents systèmes sémiotiques mobilisés que j'ai rapidement analysés précédemment participent tous à (et de...) cette paraphrase générale: ils digitalisent (un devenir-langage) tous peu ou prou les $\mathrm{S} / \mathrm{C}$ et les disséminent en éléments discrets difficiles à synthétiser et ce, d'autant plus que d'autres sémioses (gestion, identification du matériel, des TDP, des supports, des tâches, des activités, du rythme des élèves, du temps didactique, des interactions, etc.) participent à toute séance de classe et viennent nécessairement, et continuellement, perturber celles des paraphrases emboitées mentionnées ci-dessus. Or, ces successions de paraphrases (ou de "traductions ») sont déjà chacune, pour elle-même, un système sémiotique et/ou sémiologique spécifique. Ces systèmes (ceux que j'ai décrits plus haut) ne sont jamais considérés en tant qu'ensemble organisé mais comme des contributions à un ensemble didactique qui les subsume, en l'occurrence l'étude du Loup et l'Agneau.

L'imbrication des diverses désignations de $\mathrm{S} / \mathrm{C}$ en tant que systèmes sémiotiques et dans les systèmes sémiotiques qui les portent (essentiellement de types scripturaux, Lahire, 1993) peut donc être vue comme l'imbrication d'autant de sous-systèmes hétérogènes contributifs à l'intégration progressive dans un ensemble textuel cohérent. Or, toute lecture procède par emboitements successifs des divers éléments thématiques du texte lu, construisant ainsi des contextes de plus en plus élargis pouvant aller, via le texte considéré, jusqu'à l'ensemble du monde. Ces contextes peuvent à leur tour être vus comme autant de constructions sémiotiques et sémiologiques à partager, sinon partagées. Mais au-delà de ces constructions sémiotiques et sémiologiques, c'est bien leur hétérogénéité, leurs liens le plus souvent indirects et leur complémentarité postulée qui complexifient la situation didactique observée. Les élèves doivent en effet effectuer une sorte de sémiose des différentes sémioses intermédiaires réalisées, c'est-à-dire prélever dans celles-ci les éléments significatifs pour l'objectif poursuivi, lequel, je le rappelle, ne peut être connu des élèves avant d'être atteint (réticence). Autrement dit, ils doivent mesurer la pertinence des sémioses en jeu selon cet objectif et donc, pour l'exemple qui nous intéresse ici, déterminer comment et en quoi ces sémioses participent à la lecture de la fable considérée (connaissances), voire à l'apprentissage de la lecture, éventuellement littéraire (savoirs). Réussir à l'école, apprendre, c'est alors réussir simultanément et au bon moment, d'une part, à effectuer les " bonnes » sémioses et, d'autre part, à inhiber, les sémioses "indésirables ». Travail d'autant plus difficile que les élèves sont loin de l'univers sémiotique de l'école. Il faudra bien entendu revenir sur ces conclusions 
beaucoup trop rapides et générales. La présente contribution n'avait au mieux pour objectif, je l'ai déjà écrit, que de proposer, sous l'égide je l'espère bienveillante, de la sémiotique et de la sémiologie, une voie commune d'analyse.

\section{BIBLIOGRAPHIE}

AGAMBEN, G. (2009) : Signatura rerum. Sur la méthode, Paris, Vrin.

BILLETER, J.-F. (2012) : Un paradigme, Paris, Allia.

BONNERY, S. (2007) : Comprendre l'échec scolaire. Élèves en difficultés et dispositifs pédagogiques, Paris, Éd. La Dispute (coll. « L'enjeu scolaire »).

BRousseau, G. (1998) : Théorie des situations didactiques, Grenoble, Éd. La Pensée sauvage.

CARTWRIGHT, N. (1999) : The Dappled World: A Study of the Boundaries of Science, Cambridge, Cambridge University Press.

CITTON, Y. (2007) : Lire, interpréter, actualiser - pourquoi des études littéraires ?, Paris, Amsterdam. DAUnAY, B. (2002a) : Éloge de la paraphrase, Saint-Denis, Presses universitaires de Vincennes. - (2002b) : La paraphrase dans l'enseignement du français, Berne, P. Lang.

DELEUZE, G. (1996 [1988]) : L’Abécédaire de Gilles Deleuze. Entretiens avec Claire Parnet, Paris, Arte Éd. DELEUZE, G. \& GUATTARI, F. (1991) : Qu'est-ce que la philosophie ?, Paris, Éd. de Minuit. DEsCOMBES, V. (2004) : Le complément de sujet. Enquête sur le fait d'agir soi-même. Paris, Gallimard (coll. « NRF Essais »).

DEWEY, J. (2006 [1967]) : Logique. La théorie de l'enquête, trad. de l'américain, Paris, PUF.

Eco, U. (1985 [1979]) : Lector in fabula, trad. de l'italien, Paris, Grasset (coll. « Figures »).

FISH, S. (2007 [1980]) : Is There a Text in This Class? The Authority of Interpretative Communities, Cambridge, Harvard University Press.

FOUCAUlt, M. (1969) : L'archéologie du savoir, Paris, Gallimard.

- (1971) : L'ordre du discours, Paris, Gallimard.

GIBSON, J. J. (1986 [1979]) : The Ecological Approach to Visual Perception, Hillsdale, Erlbaum.

GOMBERT, J. -E. (1990) : Le développement métalinguistique, Paris, Presses universitaires de France. GOODY, J. (1979 [1977]) : La raison graphique, trad. de l'anglais, Paris, Éd. de Minuit.

LAHIRE, B. (1993) : Culture écrite et inégalités scolaires. Sociologie de "l'échec scolaire » à l'école primaire, Lyon, Presses universitaires de Lyon.

LAMBERT, F. (2013) : Je sais bien, mais quand même. Essai pour une sémiotique des images et de la croyance, Paris, Éd. Non Standard.

LEFEUVRE, L. (2008) : Savoirs, dispositifs d'actualisation de savoirs et gestes d'enseignement, master EAD, sous la dir. de G. Sensevy. 
LE HIR, V. (2012) : Un dispositif de différenciation par anticipation : comprendre-interpréter un texte littéraire au CE1, mémoire de master $1 \mathrm{EAD}$, sous la dir. de D. Forest \& L. Maisonneuve, Rennes, université de Rennes 2/IUFM de Bretagne/CREAD.

- (2013) : Un dispositif didactique entre anticipation et diffusion: Deux systèmes stratégiques pour un même contrat didactique, mémoire de master $2 \mathrm{EAD}$, sous la dir. de D. Forest \& L. Maisonneuve, Rennes, université de Rennes 2/ESPÉ de Bretagne/ CREAD.

LESSING, G. E. (2008 [1759]) : Traité sur la fable, Paris, Vrin.

MAISONNEUVE, L. (2010) : Lire et apprendre à lire à l'école primaire : la question du texte littéraire, habilitation à diriger des recherches sous la dir. de G. Sensevy.

MARGOLINAS, C. \& LAPARRA, M. (2011) : « Des savoirs transparents dans le travail des professeurs à l'école primaire », in : J.-Y. Rochex \& J. Crinon (éds), La construction des inégalités scolaires, Rennes, PUR, p. 19-32.

PELTIER, M.-L. (dir.) (2004) : Dur d'enseigner en ZEP, Grenoble, Éd. La Pensée sauvage.

RASTIER, F. (2003 [1999]) : De la signification au sens - Pour une sémiotique sans ontologie, trad. de l'italien (en ligne : http://www.revue-texto.net/Inedits/Rastier/Rastier_Semiotiqueontologie.html).

ROCHEIX, J.-Y. \& CRINON, J. (2011) : La construction des inégalités scolaires. Au cœur des pratiques et des dispositifs d'enseignement, Rennes, PUR (coll. « Paideia »).

SCHNEUWLY, B. (2000) : « Les outils de l'enseignant - Un essai didactique », Repères, 22, p. 23.

SCHNEUWLY, B. \& DOLZ, J. (2009) : Des objets enseignés en classe de français, Rennes, PUR

(coll. « Paideia »).

SENSEVY, G. (2008) : « Le travail du professeur pour la théorie de l'action conjointe en didactique : une activité située? ", Recherche et formation, 57, p. 39-50.

- (2011) : Le sens du savoir, Bruxelles, De Boeck (coll. « Perspectives en éducation \& formation »).

SENSEVy, G. \& MERCIER, A. (2007) : Agir ensemble, Rennes, PUR (coll. « Paideia »).

SENSEVY, G. \& SANTINI, J. (2006) : « Modélisation : une approche épistémologique », Aster, 43, p. $163-187$.

SOLER, C. (2009) : Lacan, l'inconscient réinventé, Paris, PUF.

TERWAGNE, S. \& VANESSE, M. (2008) : Le récit à l'école maternelle - Lire, jouer, raconter des histoires, Bruxelles : De Boeck.

WITTGENSTEIN, L. (1965) : De la certitude, Paris, Gallimard.

\section{NOTES}

1. Ce séminaire a pour objet la description de la pratique professionnelle des enseignants. Sa composition est pluricatégorielle: étudiants de master, doctorants, professeurs des écoles, formateurs d'enseignants, personnels d'encadrement de l'éducation nationale, enseignantschercheurs. La diffusion du travail effectué se fait par l'intermédiaire de mémoires (master, thèse...), de communications et d'articles.

2. La composition de ce groupe, comme celui du séminaire présenté plus haut, est pluricatégorielle : conseiller pédagogique, enseignants, maitres-formateurs, étudiants de master, doctorants, formateurs, enseignants-chercheurs. Il a pour objectif la description et l'analyse de 
séances de classe. Sa méthodologie repose sur une conception collégiale de l'ensemble du travail : élaboration des séances et des dispositifs didactiques, une étude et une retranscription des films de celles-ci et sur l'élaboration d'ingénieries didactiques. Les fables de La Fontaine ont servi de point d'ancrage à l'ensemble des travaux. Le travail de cette année 2013-2014 porte sur l'outil TPC (texte, paraphrase, commentaire) utilisé dans une classe lors de l'étude du Loup et l'Agneau. Ce tableau sera présenté plus bas.

3. Sémiotique : étude des systèmes de signes et de significations linguistiques. Sémiologie : étude des systèmes de signes et de significations (à l'œuvre dans les champs de la création et de la communication).

4. Master EAD : enseignement, apprentissages, didactiques.

5. Le Hir, V. (2012) : Un dispositif de différenciation par anticipation: comprendre-interpréter un texte littéraire au CE1 et Le Hir, V. (2013): Un dispositif didactique entre anticipation et diffusion: deux systèmes stratégiques pour un même contrat didactique, mémoires de $1^{\mathrm{re}}$ et $2^{\mathrm{e}}$ années de master EAD sous la direction de D. Forest et L. Maisonneuve.

6. Pour une définition du «Réel» et de la "Réalité » avec une majuscule, voir C. Soler (2009). Pour Lacan et Soler, le Réel peut être considéré comme le «monde » ou la "vie». Il est incontournable : nous sommes dans le Réel et nous sommes le Réel. La Réalité n'est qu'une partie du Réel, celle à laquelle nous avons accès (par exemple par le symptôme), celle que nous médiatisons par les langages. Lacan a formé pour cela le concept de "lalangue ", à la fois «corps» et «langue/langage». Si J.-F. Billeter (2012: 28-33) appelle "monde» la Réalité et « réalité » le «Réel ", ses analyses sont très proches de celles de Lacan et de Soler : "Je fais à dessein une distinction entre monde et réalité. J'entends par monde l'ensemble des choses parmi lesquelles nous vivons, créées par l'objectivation et le langage au sein de la réalité par réalité tout ce qui existe en nous et hors de nous, indépendamment et au-delà des formes créées par l'objectivation et le langage » (ibid. : 30).

7. En se sens, un « savoir, c'est ce dont on peut parler dans une pratique discursive qui se trouve par là spécifiée : le domaine constitué par les différents objets qui acquerront ou non un statut scientifique [...], l'espace dans lequel le sujet peut prendre position pour parler des objets auxquels il a affaire dans son discours [...] le champ de coordination et de subordination des énoncés où les concepts apparaissent, se définissent, s'appliquent et se transforment [...] enfin, un savoir se définit par des possibilités d'utilisation et d'appropriation offertes par le discours » (Foucault, 1969:238). Ici, si M. Foucault ne fait la différence que j'opère entre savoir et connaissance, il souligne le lien en quelque sorte consubstantiel du savoir, savoir selon mon acception, et d'une forme, d'une formation, discursive.

8. Voir aussi plus bas la description de ce que J.-E. Gombert (1992) appelle les stades " épilinguistique » et « métalinguistique ».

9. Même s'il est permis, bien entendu, de considérer cette contextualisation initiale comme une première institutionnalisation. Il n'y a pas de commencement absolu, pas de régression à l'infini, mais des "posés » successifs, des épistémès : «Par épistémè, on entend, en fait, l'ensemble des relations pouvant unir, à une époque donnée, les pratiques discursives qui donnent lieu à des figures épistémologiques, à des sciences, éventuellement à des systèmes formalisés » (Foucault, 1969 : 250). Il poursuit en précisant ces relations comme une «mode » selon lequel des passages s'opèrent, passages vus comme autant de « seuils » qui entrent en contact, se frottent en quelque sorte. On s'interrogera ainsi davantage sur les/aux conditions de possibilité, sur les/aux conditions de surgissement, d'une pratique discursive, d'une épistémè, qu'à son origine. On privilégiera donc plutôt l'immanence (ou "plan d'immanence », comme ensemble d'énoncés liés par une pratique, selon les termes de Deleuze et Guattari, 1991) que la transcendance. Sans aucun doute, le concept de paradigme, comme l'entend G. Agamben (2009), pourrait prétendre se substituer à ces diverses dénominations : «Le paradigme n'est jamais donné, mais s'engendre et se produit [...] au moyen d'un placer à côté, un joindre ensemble et, surtout, un montrer et un 
exposer. [...] La relation paradigmatique ne s'établit donc pas simplement entre les objets sensibles singuliers, ni entre ceux-ci une règle générale, mais d'abord entre la singularité (qui devient ainsi paradigmatique) et son exposition (c'est-à-dire son intelligibilité)» (ibid. : 25-26). Voir aussi sur le même thème : V. Descombes (2004 : 221 sqq) : «Par exemple, nous n'étudions ni un "phénomène" du temps, ni les phénomènes de la conscience du temps, mais plutôt l'usage de petits mots tels que "avant", "après", "en même temps", "longtemps", etc. ».

10. $S$ pour séance, 4 pour numéro de la séance, TDP pour tour de parole, 195 pour numéro d'ordre du TDP dans la séance.

11. «Car vous ne m'épargnez guère,

Vous, vos bergers, et vos chiens.

On me l'a dit : il faut que je me venge. » (J. de La Fontaine, Le Loup et l'Agneau, v. 24-26).

12. L'affordance est la capacité d'un objet, ici d'un texte, à suggérer sa propre utilisation, autrement dit la possibilité offerte à un utilisateur de s'en emparer de l'objet sans recourir à une médiation extérieure à celui-ci et à lui-même. Certains emploient aussi en l'occurrence le terme de convivialité, voire d'objets conviviaux.

\section{RÉSUMÉS}

Á partir du travail réalisé par le Groupe ESPÉ de recherche et de production de ressources pour l'éducation et la formation (GERPREF) «Fables et anticipation» et du séminaire Action du professeur (IUFM/ESPÉ de Bretagne), j'aimerais montrer que le sens du savoir se construit simultanément aux formes qui le manifestent. Cette contribution s'inscrit dans la continuité des travaux de G. Sensevy (2011) sur le Sens du savoir. Elle a pour objectif d'examiner les différentes sémioses à l'œuvre lors de l'étude d'un texte littéraire en classe de CE1. Le texte littéraire retenu est la fable Le Loup et l'Agneau de Jean de La Fontaine. J'analyse ici plus particulièrement comment les différentes sémioses contribuent à la lecture du texte, même et surtout lorsque celles-ci semblent éloignées de cet objectif. La perspective est également, à terme, d'essayer de construire un cadre d'analyse homogène d'obédience sémiotique. Seront ainsi successivement analysés : le lexique et le métalangage; le matériel, les supports, l'organisation de la classe; l'habitus, l'intertextualité, l'explicite et l'implicite textuels ; les tâches et les activités ; les ajustements, les savoirs/connaissances du professeur et des élèves.

Based on the work done by the GERPREF "Fables et anticipation" and on the seminary "Action du professeur" (IUFM/ESPE of Brittany), I would like to show that the meaning of knowledge builds itself simultaneously with the shapes that express it. This article further develops the Sensevy's work on the Sense of knowledge (2011). It aims at analyzing the action of different semiosis on a literary text during a CE1 (Year 3, $2^{\text {nd }}$ grade) class session. The chosen literary text is La Fontaine's the Wolf and the Lamb fable. In this article, I will study how different semiosis contribute to the reading of a text; especially when they do not seem to pursue this particular goal. Ultimately, my purpose is to build a homogenous framework of semiotic obedience. Thus, the following aspects will be successively analyzed: lexicon and metalanguage, equipments, media, class organization, habitus, intertextuality, the textually explicit and implicit, tasks and activities, adjustments, knowledge of the teacher and students. 
INDEX

Mots-clés : savoir, connaissance, sémiose, formations sémiotiques, lecture (littéraire),

sémiologie

Keywords : knowledge, semiosis, semiotic's organisations and developments, reading (litterary text), semiology

\section{AUTEUR}

LUC MAISONNEUVE

CREAD (EA 3875), ESPÉ de Bretagne, Université européenne de Bretagne 